\title{
DEGRADAÇÃO ENZIMÁTICA DO CORANTE DE COCHONILHA
}

M. A. BOTH ${ }^{1}$, S. FARIAS ${ }^{1}$, R. E. FRANZOI Jr ${ }^{1}$, D. TODESCATO ${ }^{1}$, S. M. A. GUELLI U. SOUZA $^{1}$, A. A. ULSON DE SOUZA ${ }^{1}$

${ }^{1}$ Universidade Federal de Santa Catarina, Departamento de Engenharia Química e Engenharia de Alimentos

E-mails para contato: augusto@enq.ufsc.br,michelly_both_23@hotmail.com

RESUMO: O efluente têxtil contém corantes sintéticos hidrolisados que podem causar um grande impacto quando descartados em ambientes aquáticos. Os corantes naturais tornam o processo de tingimento uma rota de menor impacto ambiental, principalmente porque são produzidos a partir de plantas e animais. O objetivo deste trabalho é degradar o corante carmim de cochonilha utilizando a enzima Horseradish peroxidase (HRP). Os efeitos das principais condições que influenciam o processo, como $\mathrm{pH}$, concentração de corante, enzima e peróxido de hidrogênio, foram estudados. A otimização do processo foi feita utilizando a metodologia da superfície de resposta e posteriormente foi realizado um segundo planejamento $2^{4}$ completo para obtenção da condição ótima de degradação. As concentrações de enzima e peróxido de hidrogênio, corante e $\mathrm{pH}$ foram determinadas. Foi utilizado um limite de confiança de $95 \%$ foi obtido um ajuste ao modelo de $89 \%$.

\section{INTRODUÇÃO}

Peroxidases (EC 1.11.1.7) são um grupo de enzimas oxidorredutases que requerem peróxido de hidrogênio para degradar uma variedade de substratos orgânicos e inorgânicos (SI; CUI, 2013). Existem vários trabalhos reportados na literatura sobre o uso de peroxidases de diversos tipos de fontes vegetais para remover corantes reativos, como peroxidase do nabo (CORREA et al., 2012), peroxidase da soja (MARCHIS et al., 2011), peroxidase da batata (LONCAR et al., 2012), peroxidase da raiz forte (JIANG et al., 2014). Atualmente destaca-se alguns trabalhos que utilizam enzimas como a Horseradish peroxidase para remover corantes naturais como corante da flor de hibisco e suco de romã (GUELLI, U. SOUZA. S.M.A; FORGIARINI, E; ULSON de SOUZA A.A., 2007, ABDEL-ATY et al., 2013).

Corantes naturais foram os corantes mais utilizados até o meio do século XIX (COLOMBINI et al., 2007). Com o surgimento dos corantes sintéticos que possuem características como uma grande variedade de cor, boa solidez à lavagem, facilidade de 
aplicação e consumo mínimo de energia (CHEN et al., 2011) os corantes naturais foram gradualmente substituídos. Devido a rigorosas normas ambientais houve um aumento no interesse da utilização de corantes naturais nas indústrias têxteis nos últimos anos, principalmente em respostas às reações tóxicas e alérgicas associadas aos corantes sintéticos, em diversas áreas estes corantes já foram substituídos pelos naturais (GUESMI et al., 2013; BALIARSINGH et al., 2012). Os corantes naturais possuem baixa toxicidade, são ecologicamente corretos, os derivados de plantas são extremamente abundantes e ainda podem apresentar algumas propriedades funcionais quando aplicados em têxteis como proteção antimicrobiana, propriedades desodorantes e aromáticas, entre outras propriedades que agregam valor ao produto final (UL-ISLAM et al., 2013).

O ácido carmínico, Figura 1, é um corante extraído de fêmeas dessecadas de insetos da espécie Dactylopius coccus é o principal constituinte da cochonilha sendo responsável por seu poder tintorial. Quimicamente é um composto orgânico derivado da antraquinona, solúvel em água e com boa estabilidade a luz (BORGES et al., 2012).

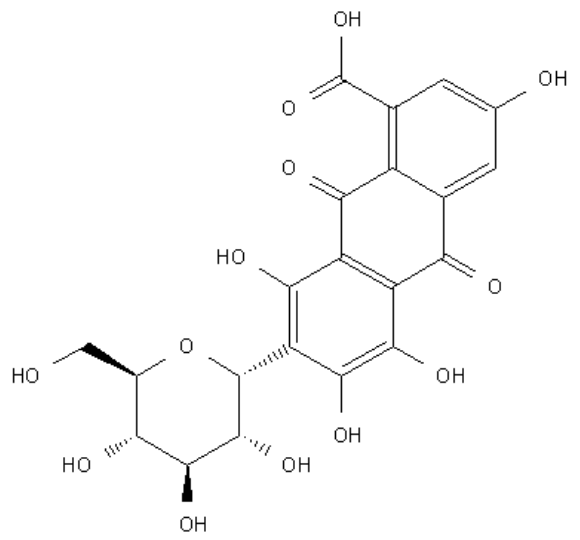

Figura 1 - Ácido carmínico.

O objetivo deste trabalho é analisar a degradação do corante de cochonilha utilizando enzima peroxidase principalmente com objetivo de utilizar esse corante na indústria têxtil. Esta análise será realizada através de um planejamento estrela avaliando os principais fatores que afetam a degradação enzimática como quantidade enzima, peróxido de hidrogênio, corante e $\mathrm{pH}$.

\section{MATERIAIS E MÉTODOS}

\subsection{Reagentes e Enzimas}

A enzima Horseradish peroxidase utilizada neste trabalho foi gentilmente cedida pela empresa Toyobo. O corante de carmim de cochonilha utilizado neste trabalho foi adquirido na empresa Biocarmim, sendo uma solução preparada com hidróxido de potássio, água e carmim roxo. 


\subsection{Planejamento Experimental}

No estudo de degradação do corante de cochonilha foram analisados quatro fatores (concentração de enzima, peróxido de hidrogênio, corante e pH) bem como suas possíveis interações.

O planejamento realizado foi do tipo estrela com triplicata do ponto central e pontos axiais. Após a definição do tipo de planejamento os níveis de variação foram definidos. As variáveis estudadas e seus respectivos níveis de variação são mostrados na Tabela 1 .

Tabela 1- Fatores e níveis estudados durante o Planejamento Experimental

\begin{tabular}{cccccc}
\hline Variáveis & \multicolumn{5}{c}{ Níveis de estudo } \\
\hline $\begin{array}{c}\text { Concentração de } \\
\text { Enzima U/ml }\end{array}$ & 576,9 & 1153,9 & 1730,8 & 2307,7 & 2884,6 \\
$\begin{array}{c}\text { Concentração de } \\
\mathrm{H}_{2} \mathrm{O}_{2} \mu \mathrm{M}\end{array}$ & 150,0 & 200,0 & 250,0 & 300,0 & 350,0 \\
$\begin{array}{c}\text { Concentração de } \\
\text { corante mg/l } \\
\text { pH }\end{array}$ & 200,0 & 400,0 & 600,0 & 800,0 & 1000,0 \\
\hline
\end{tabular}

\subsection{Determinação da atividade enzimática}

A atividade inicial do extrato foi determinada segundo a metodologia modificada de Wu et al. (1997). Para a determinação da atividade foram utilizados $255 \mu \mathrm{L}$ de solução de ABTS $20 \mathrm{mM}, 255 \mu \mathrm{L}$ de peróxido de hidrogênio $\left(\mathrm{H}_{2} \mathrm{O}_{2}\right) 10 \mathrm{mM}, 300 \mu \mathrm{L}$ de extrato enzimático $1 \mathrm{mM}, 2190 \mu \mathrm{L}$ de tampão fosfato $\mathrm{pH} 6\left(0,05 \mathrm{molL}^{-1}\right)$.

Todas as soluções foram preparadas em tampão fosfato $\mathrm{pH}$. A reação foi mantida a $30^{\circ} \mathrm{C}$ em banho termostatizado por 10 min e em seguida acompanhada durante 5 min, em um espectrofotômetro a $420 \mathrm{~nm}$. A atividade enzimática (A) foi calculada conforme a Equação 1 .

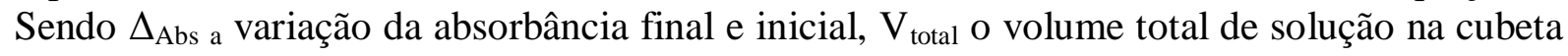
em $\mathrm{mL}, \mathrm{V}_{\text {extrato }} \mathrm{O}$ volume de extrato enzimático utilizado na reação em $\mathrm{mL}, \varepsilon$ o coeficiente de extinção molar do ABTS $\left(\varepsilon_{420 \mathrm{~nm}}=3,6 \times 10^{4} \mathrm{M}^{-1} . \mathrm{cm}^{-1}\right)$ e $\mathrm{t}$ o tempo em minutos.

$$
\mathrm{A}(\mathrm{U} / \mathrm{mL})=\frac{\Delta_{\text {Abs. }} . \mathrm{V}_{\text {total. }} 10^{6}}{\varepsilon_{\text {ABTS.VExtrato. }} \text { t }}
$$

\subsection{Degradação enzimática do corante de cochonilha}

As reações de degradação enzimática foram conduzidas conforme metodologia modificada de Silva et al., (2012). Foram utilizados $0,4 \mathrm{~mL}$ de solução de $\mathrm{H}_{2} \mathrm{O}_{2}, 0,1 \mathrm{~mL}$ de extrato enzimático, $1,5 \mathrm{~mL}$ de corante e tampão citrato ou fosfato $0,05 \mathrm{~mol} / \mathrm{L}(1,2 \mathrm{~mL})$. As soluções de $\mathrm{H}_{2} \mathrm{O}_{2}$ e de extrato enzimático foram preparadas em tampão nos diferentes. Nos controles reacionais foram empregados todos os reagentes com exceção do $\mathrm{H}_{2} \mathrm{O}_{2}$ que foi 
substituído por tampão. A absorbância foi medida em $519 \mathrm{~nm}$. A porcentagem de degradação (D) foi calculada pela equação 2 .

$$
\frac{D(\%)=A b s_{\text {inicial }}-A b s_{\text {final }}}{A b s_{\text {inicial }}} .100
$$

\section{RESULTADOS E DISCUSSÃO}

Na Tabela 2, pode ser observada as descolorações obtidas para o corante de cochonilha, a maior descoloração de $33 \%$ foi obtida quando se utilizou 2307, $70 \mathrm{U} / \mathrm{mL}$ de enzima, $200 \mathrm{mM}$ de peróxido de hidrogênio, $800 \mathrm{mg} / \mathrm{L}$ de corante e $\mathrm{pH}$ 4,0. Abdel-Aty et al.,(2013) realizaram a degradação de vários corantes reativos e naturais pelas enzimas HRP e Ficus sycomorus latex peroxidase, foi utilizado um corante azo-carmim e para este corante foram obtidas degradações de 30-33\%.

Tabela 2- Descolorações obtidas para o corante de cochonilha

\begin{tabular}{cccccc}
\hline Ensaio & $\begin{array}{c}\text { Enzima } \\
\text { U/mL }\end{array}$ & $\begin{array}{c}\mathbf{H}_{\mathbf{2}} \mathbf{O}_{\mathbf{2}} \\
\mathbf{m M}\end{array}$ & $\begin{array}{c}\text { Corante } \\
\mathbf{m g} / \mathbf{L}\end{array}$ & $\mathbf{p H}$ & $\begin{array}{c}\text { Descoloração } \\
(\mathbf{\%})\end{array}$ \\
\hline $\mathbf{1}$ & -1 & -1 & -1 & -1 & 29 \\
$\mathbf{2}$ & -1 & -1 & -1 & 1 & 5 \\
$\mathbf{3}$ & -1 & -1 & 1 & -1 & 21 \\
$\mathbf{4}$ & -1 & -1 & 1 & 1 & 7 \\
$\mathbf{5}$ & -1 & 1 & -1 & -1 & 24 \\
$\mathbf{6}$ & -1 & 1 & -1 & 1 & 6 \\
$\mathbf{7}$ & -1 & 1 & 1 & -1 & 24 \\
$\mathbf{8}$ & -1 & 1 & 1 & 1 & 6 \\
$\mathbf{9}$ & 1 & -1 & -1 & -1 & 31 \\
$\mathbf{1 0}$ & 1 & -1 & -1 & 1 & 12 \\
$\mathbf{1 1}$ & 1 & -1 & 1 & -1 & 33 \\
$\mathbf{1 2}$ & 1 & -1 & 1 & 1 & 6 \\
$\mathbf{1 3}$ & 1 & 1 & -1 & -1 & 28 \\
$\mathbf{1 4}$ & 1 & 1 & -1 & 1 & 1 \\
$\mathbf{1 5}$ & 1 & 1 & 1 & -1 & 29 \\
$\mathbf{1 6}$ & 1 & 1 & 1 & 1 & 6 \\
$\mathbf{1 7}$ & -2 & 0 & 0 & 0 & 9 \\
$\mathbf{1 8}$ & 2 & 0 & 0 & 0 & 16 \\
$\mathbf{1 9}$ & 0 & -2 & 0 & 0 & 12 \\
$\mathbf{2 0}$ & 0 & 2 & 0 & 0 & 14 \\
$\mathbf{2 1}$ & 0 & 0 & -2 & 0 & 15 \\
$\mathbf{2 2}$ & 0 & 0 & 0 & 0 & 12 \\
$\mathbf{2 3}$ & 0 & 0 & 0 & -2 & 27 \\
$\mathbf{2 4}$ & 0 & 0 & 0 & +2 & 4 \\
$\mathbf{2 5}$ & 0 & 0 & 0 & 0 & 12 \\
$\mathbf{2 6}$ & 0 & 0 & 0 & 0 & 12 \\
$\mathbf{2 7}$ & 0 & 0 & 0 & 0 & 13 \\
\hline
\end{tabular}


Por meio dos resultados obtidos na Tabela 2 realizou-se os cálculos dos efeitos apresentados na Tabela 3. Analisando-se a Tabela 3 é possível observar que somente o fator linear do corante não foi significativo, todos os demais fatores tanto linear como quadrático foram significativos. Também, foi observado que as interações entre enzima e peróxido de hidrogênio, enzima e corante, peróxido de hidrogênio e $\mathrm{pH}$ e corante e $\mathrm{pH}$ não influenciaram a descoloração do corante de cochonilha a um nível de confiança de $95 \%$.

Tabela 3 - Cálculo dos efeitos e respectivos índices estatísticos

\begin{tabular}{ccccccc}
\hline & & & & \multicolumn{2}{c}{ Limite de confiança } \\
\cline { 6 - 7 } & Efeito & $\begin{array}{c}\text { Erro } \\
\text { padrão }\end{array}$ & $\begin{array}{c}\text { t } \\
\text { Student }\end{array}$ & $\mathbf{p}$ & $\mathbf{- 9 5 \%}$ & $\mathbf{+ 9 5 \%}$ \\
\hline Média/interações & $\mathbf{1 2 , 3 3 3}$ & $\mathbf{0 , 3 3 3}$ & $\mathbf{3 7 , 0 0 0}$ & $\mathbf{0 , 0 0 0}$ & $\mathbf{1 0 , 8 9 9}$ & $\mathbf{1 3 , 7 6 7}$ \\
(1)Enzima (L) & $\mathbf{3 , 1 6 6}$ & $\mathbf{0 , 2 3 5}$ & $\mathbf{1 3 , 4 3 5}$ & $\mathbf{0 , 0 0 5}$ & $\mathbf{2 , 1 5}$ & $\mathbf{4 , 1 9}$ \\
Enzima (Q) & $\mathbf{1 , 1 2 5}$ & $\mathbf{0 , 2 5 0}$ & $\mathbf{4 , 5 0 0}$ & $\mathbf{0 , 0 4 6}$ & $\mathbf{0 , 0 5}$ & $\mathbf{2 , 1 0}$ \\
(2) H $_{2} \mathbf{O}_{2}$ (L) & $\mathbf{- 1 , 3 3 3}$ & $\mathbf{0 , 2 3 5}$ & $\mathbf{- 5 , 6 5 7}$ & $\mathbf{0 , 0 2 9}$ & $\mathbf{- 2 , 3 4}$ & $\mathbf{- 0 , 3 2}$ \\
H2 $\mathbf{O}_{\mathbf{2}}$ (Q) & $\mathbf{1 , 3 7 5}$ & $\mathbf{0 , 2 5 0}$ & $\mathbf{5 , 5 0 0}$ & $\mathbf{0 , 0 3 1}$ & $\mathbf{0 , 3 0}$ & $\mathbf{2 , 4 6}$ \\
(3) Corante (L) & $-0,833$ & 0,235 & $-3,535$ & 0,071 & $-1,85$ & 0,19 \\
Corante (Q) & $\mathbf{1 , 6 2 5}$ & $\mathbf{0 , 2 5 0}$ & $\mathbf{6 , 5 0 0}$ & $\mathbf{0 , 0 2 3}$ & $\mathbf{0 , 5 5}$ & $\mathbf{2 , 7 1}$ \\
(4) pH (L) & $\mathbf{- 1 8 , 0 0 0}$ & $\mathbf{0 , 2 3 5}$ & $\mathbf{- 7 6 , 3 6 8}$ & $\mathbf{0 , 0 0 0}$ & $\mathbf{- 1 9 , 0 0}$ & $\mathbf{- 1 6 , 9 9}$ \\
pH (Q) & $\mathbf{2 , 6 2 5}$ & $\mathbf{0 , 2 5 0}$ & $\mathbf{1 0 , 5 0 0}$ & $\mathbf{0 , 0 0 9}$ & $\mathbf{1 , 5 5}$ & $\mathbf{3 , 7 0}$ \\
1L e 2L & $-2,000$ & 0,288 & $-6,928$ & 0,0202 & $-3,24$ & $-0,76$ \\
1L e 3L & 1,000 & 0,288 & 3,464 & 0,074 & $-0,24$ & 2,24 \\
1L e 4L & $\mathbf{- 2 , 7 5 0}$ & $\mathbf{0 , 2 8 8}$ & $\mathbf{- 9 , 5 2 6}$ & $\mathbf{0 , 0 1 0}$ & $\mathbf{- 3 , 9 9}$ & $\mathbf{- 1 , 5 1}$ \\
2L e 3L & $\mathbf{2 , 0 0 0}$ & $\mathbf{0 , 2 8 8}$ & $\mathbf{6 , 9 2 8}$ & $\mathbf{0 , 2 0 2}$ & $\mathbf{0 , 7 6}$ & $\mathbf{3 , 2 4}$ \\
2 L e 4 L & $-0,250$ & 0,288 & $-0,866$ & 0,478 & $-1,49$ & 0,99 \\
3L e 4L & 0,750 & 0,288 & 2,598 & 0,122 & $-0,49$ & 1,99 \\
\hline
\end{tabular}

Na Figura 2, pode-se observar a curva de nível correspondente à superfície de resposta gerada pelo modelo.

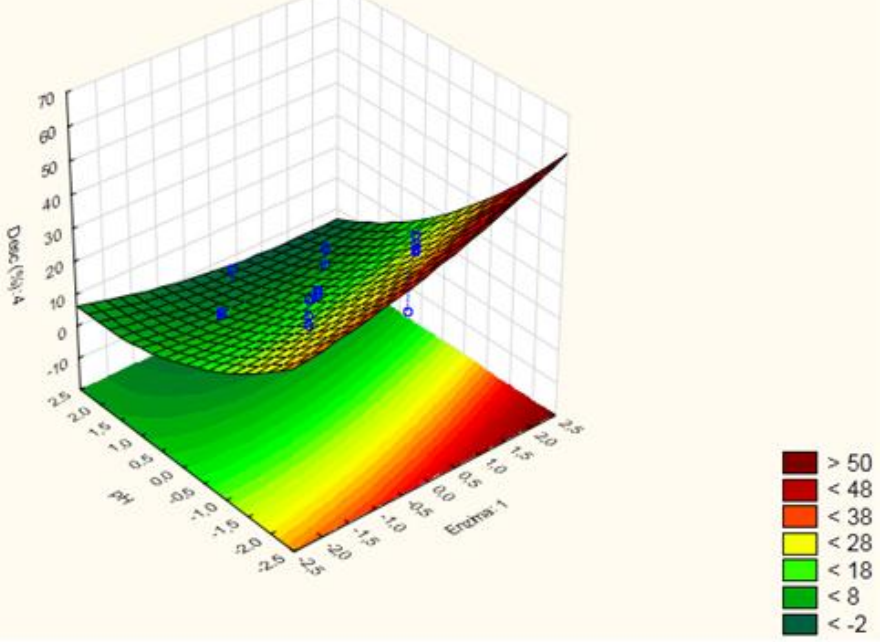

Figura 2- Curva de superfície para os fatores enzima e pH em relação a resposta descoloração do corante. 
$\mathrm{O}$ fator $\mathrm{pH}$ é negativo e o fator enzima é positivo, a interação dos dois é negativa. $\mathrm{O}$ sinal negativo do efeito da interação enzima e pH demonstra que a descoloração de corante tem comportamento inverso à variação desta interação, ou seja, um acréscimo no valor do fator $\mathrm{pH}$ ocasiona um decréscimo no valor de descoloração de corante. Conforme apresentado na Figura 2, pode-se observar que um aumento na concentração de enzima e a diminuição do $\mathrm{pH}$ ocorre um aumento na descoloração do corante pela enzima HRP.

Guelli; Forgiarini e Ulson de Souza (2007) realizaram a descoloração do corante turquesa remazol G $133 \%$ utilizando a enzima Horseradish peroxidase a máxima remoção foi obtida em pH 4, 59\% de descoloração. Em geral descoloração de corante com enzima peroxidase o pH ótimo de descoloração sempre está na faixa encontrada por Guelli; Forgiarini e Ulson de Souza (2007) 4,0-5,5. Khan e Husain (2007) realizaram a descoloração de corantes reativos com enzima peroxidase e a máxima descoloração foi encontrada em $\mathrm{pH}$ ácido entre 3,0-4,0. Abdel-Aty et al. (2013) realizaram a descoloração dos corantes naturais de hibisco e punica granatum, sendo que os máximos de descoloração de 80 e $82 \%$, respectivamente, foram obtidas em $\mathrm{pH}$ 5,5. O modelo de descoloração de corante pela enzima HRP é representado na Equação 1.

$\mathrm{D}=12,333+1,583$ [enzima] $+0,562[\text { enzima }]^{2}-0,666\left[\mathrm{H}_{2} \mathrm{O}_{2}\right]$

$+0,687\left[\mathrm{H}_{2} \mathrm{O}_{2}\right]^{2}+0,812[\text { corante }]^{2}-9,000[\mathrm{pH}]+1,312[\mathrm{pH}]^{2}$

$-1,000$ [Enzima]. $\left[\mathrm{H}_{2} \mathrm{O}_{2}\right]-1,375$ [Enzima].[pH]

$+1,000\left[\mathrm{H}_{2} \mathrm{O}_{2}\right]$. [Corante].

Na Tabela 4 é apresentada a análise de variância (ANOVA) do modelo obtido.

Tabela 4- Análise de variância para a degradação do corante de cochonilha

\begin{tabular}{cccccc}
\hline $\begin{array}{c}\text { Fonte de } \\
\text { variação }\end{array}$ & $\begin{array}{c}\text { Soma } \\
\text { Quadrática }\end{array}$ & $\begin{array}{c}\text { Graus de } \\
\text { Liberdade }\end{array}$ & $\begin{array}{c}\text { Média } \\
\text { Quadrática }\end{array}$ & $\begin{array}{c}\text { F } \\
\text { calculado }\end{array}$ & $\begin{array}{c}\text { F } \\
\text { tabelado }\end{array}$ \\
\hline Regressão & 2128,39 & 14 & 152,02 & 7,49 & 2,53 \\
Resíduos & 243,61 & 12 & 20,30 & & \\
Falta de Ajuste & 242,83 & 10 & 24,28 & 73,58 & 4,10 \\
Erro puro & 0,67 & 2 & 0,33 & & \\
Total & 2372,00 & 26 & & & \\
\hline
\end{tabular}

Variação explicada: 89,73

Variação máxima explicável: 99,97

Pode-se observar na Tabela 4, que a soma quadrática dos erros é muito pequena, o que nos indica que o modelo é bom. Pelos valores de $\mathrm{F}$ calculados para o resíduos em relação à regressão que é quase 3 vezes maior que o valor de $\mathrm{F}$ tabelado mostrando que a regressão é significativa, podendo ser usada para fins de predição. O F calculado para o erro puro em relação a falta de ajuste é bem maior do que o F tabelado, indicando uma falta de ajuste ao modelo. Os valores de resíduos se comparado à regressão não são significativos. $\mathrm{O}$ erro puro 
encontrado é praticamente desprezível. O valor da variação máxima explicável, que é de $99,97 \%$ é alto frente à variação explicada.

\section{CONCLUSÃO}

Para o corante natural de cochonilha foi obtido um valor máximo de degradação em torno de $31-33 \%$. Pode-se concluir que as faixas de concentração de enzima deveriam ser aumentadas e o pH mantido ácido para aumentar a degradação desse corante, segundo o modelo estatístico. Foi obtido um ajuste ao modelo de $89 \%$ para um limite de confiança de $95 \%$.

\section{REFERÊNCIAS}

ABDEL-ATY, A.; HAMED, M. B.; FAHMY, A. S.; MOHAMED, S. A. Comparison of the potential of Ficus sycomorus latex and horseradish peroxidases in the decolorization of synthetic and natural dyes. J. Genet. Eng. Biotechnol., v. 11, p. 95-102, 2013.

BALIARSINGH, S.; PANDA, A. K.; TRUPTI, J.; DAS, N. B. Exploring sustainable technique on natural dye extraction from native plants for textile: identification of colourants, colourimetric analysis of dyed yarns and their antimicrobial evaluation. J. Cleaner Prod., v. 37, p. 257-264, 2012.

BORGES, M. E.; TEJERA, R. L.; DIAZ, L.; ESPARZA, P.; ILBANEZ, E. Natural dyes extraction from cochineal (Dactylopius coccus). New extraction methods. Food Chem., v. 132, p. 1855-1860, 2012.

CHEN, G.; HUANG. M. H.; CHEN, L.; CHEN, D. H. A batch decolorization and kinetic study of reactive black 5 by a bacterial strain Enterobacter sp. GY-1. Int. Biodeter. Biodeg., v. 65, p. 790-796, 2011.

COLOMBINI, M. P.; ANDREOTTI, A.; BARALDI, C.; DEGANO, I.; LUCEJKO, J. Colour fading in textiles: A model study on the decomposition of natural dyes. Microchem. J., v. 85, p. 174-182, 2007.

GUELli U. SOUZA, S. M. A; FORGIARINI, E.; ULSON de SOUZA, A. A. Toxicity of textile dyes and their degradation by the enzyme Horseradish peroxidase (HRP). J. Hazard. Mater., v. 147, p. 1073-1078, 2007.

GUESMI, A.; HAMADI, N. B.; SAKLI, F. Sonicator dyeing of modified acrylic fabrics with indicaxanthin natural dye. Ind. Crop. Prod., v. 42, p. 63- 69, 2013. 
JIANG, Y.; TANG, W.; GAO, J.; ZHOU, L.; HE, Y. Immobilization of horseradish peroxidase in phospholipid-templated titania and its applications in phenolic compounds and dye removal. Enzyme Microb. Technol., v. 55, p. 1-6, 2014. DOI: 10.1016/j.enzmictec.2013.

KHAN, A. A.; HUSAIN, Q. Decolorization and removal of textile and non-textile dyes from polluted wastewater and dyeing effluent by using potato (Solanum tuberosum) soluble and immobilized polyphenol. oxidase. Bioresource Technol., v. 98, p.1012-1019, 2007.

LONCAR, N.; JANOVI, B.; VUJCIC, M.; VUJCIC, Z. Decolorization of textile dyes and effluents using potato (Solanum tuberosum) phenoloxidase. Int. Biodeter. Biodeg., v. 72, p. 42-45, 2012.

MARCHIS, T.; AVETTA, P.; BIANCO-PREVOT, A.; FABBRI, D., VISCARDI, G.; LAURENTI, E. Oxidative degradation of Remazol Turquoise Blue G 133 by soybean peroxidase. J. Inorg. Biochem., v. 105, p. 321-327, 2011.

SI, J; CUI, B-K. A new fungal peroxidase with alkaline-tolerant, chloride-enhancing activity and dye decolorization capacity. J. Mol. Catal. B: Enzym., v. 89 p. 6-14, 2013.

WU, Y.; TAYLOR, K. E.; BISWAS, N., BEWTRA, J. K. Comparison of Additives in the Removal of Phenolic Compounds by Peroxidase Catalyzed Polymerization. Water Res., v. 31, p. 2699-2704, 1997.

UL-ISLAM, S; SHAHID, M; MOHAMMAD, F. Perspectives for natural product based agents derived from industrial plants in textile applications. A review. J. Cleaner Prod., v. 57, p. 2-18, 2013. 